\title{
Role of Sinorhizobium meliloti and Escherichia coli Long-Chain Acyl-CoA Synthetase FadD in Long-Term Survival
}

\author{
Ángel de la Cruz Pech-Canul $\left.{ }^{1,+}+{ }^{(}\right)$, Geovanny Rivera-Hernández ${ }^{1}$, Joaquina Nogales ${ }^{2, \ddagger}$, \\ Otto Geiger ${ }^{1}$, María J. Soto ${ }^{2}\left(\mathbb{D}\right.$ and Isabel M. López-Lara ${ }^{1, *}$ \\ 1 Programa de Ecología Genómica, Centro de Ciencias Genómicas, Universidad Nacional Autónoma de \\ México. Cuernavaca, Morelos, C.P. 62210, Mexico; angelpechcanul@gmail.com (Á.d.l.C.P.-C.); \\ chambolom@gmail.com (G.R.-H.); otto@ccg.unam.mx (O.G.) \\ 2 Departamento de Microbiología del Suelo y Sistemas Simbióticos. Estación Experimental del Zaidín, CSIC, \\ 18008 Granada, Spain; jnogales@noble.org (J.N.); mariajose.soto@eez.csic.es (M.J.S.) \\ * Correspondence: isabel@ccg.unam.mx; Tel.: +52-7773291703 \\ + Current address: CONACyT-Faculty of Chemical Sciences, Autonomous University of Chihuahua, \\ Chihuahua 31125, Mexico. \\ $\ddagger$ Current address: Noble Research Institute, Ardmore, OK 73401, USA.
}

Received: 15 February 2020; Accepted: 24 March 2020; Published: 26 March 2020

\begin{abstract}
FadD is an acyl-coenzyme A (CoA) synthetase specific for long-chain fatty acids (LCFA). Strains mutated in fadD cannot produce acyl-CoA and thus cannot grow on exogenous LCFA as the sole carbon source. Mutants in the fadD (smc02162) of Sinorhizobium meliloti are unable to grow on oleate as the sole carbon source and present an increased surface motility and accumulation of free fatty acids at the entry of the stationary phase of growth. In this study, we found that constitutive expression of the closest FadD homologues of S. meliloti, encoded by sma0150 and smb20650, could not revert any of the mutant phenotypes. In contrast, the expression of Escherichia coli fadD could restore the same functions as S. meliloti fadD. Previously, we demonstrated that FadD is required for the degradation of endogenous fatty acids released from membrane lipids. Here, we show that absence of a functional $\mathrm{fadD}$ provokes a significant loss of viability in cultures of E. coli and of S. meliloti in the stationary phase, demonstrating a crucial role of fatty acid degradation in survival capacity.
\end{abstract}

Keywords: long-chain acyl-CoA synthetase; free fatty acids; Sinorhizobium (Ensifer); surface motility; survival; malonyl-CoA synthetase

\section{Introduction}

Fatty acid metabolism has been studied mainly in the model organism Escherichia coli that can use long-chain fatty acids (LCFA) as sole carbon and energy source. The fatty acid degradation (Fad) pathway is responsible for the transportation, activation, and $\beta$-oxidation of LCFA (>10 carbons). These LCFA are transported into the cell by the outer membrane protein FadL and subsequently converted to their coenzyme A (CoA) thioesters by the enzyme acyl-CoA synthetase, encoded by $f a d D$ [1]. The degradation of acyl-CoAs proceeds via an inducible set of enzymes that catalyse the $\beta$-oxidative cleavage of the acyl-CoA into acetyl-CoAs. The first step in the $\beta$-oxidation cycle involves the conversion of acyl-CoA to enoyl-CoA via FadE. The remaining steps of hydration, oxidation and thiolytic cleavage in fatty acid degradation are performed by a tetrameric complex consisting of two copies each of FadA and FadB. The strains mutated in fadD cannot produce acyl-CoA and thus cannot grow on exogenous fatty acids [1]. 
Soto et al. [2] identified the fadD gene in the symbiotic nitrogen-fixing bacterium Sinorhizobium (Ensifer) meliloti GR4. Interestingly, in addition to being unable to grow on oleic acid as the sole carbon source, the mutation of $f a d D$ in $S$. melitoti GR4 resulted in multicellular swarming behaviour and defects in the establishment of symbiosis with alfalfa host plants. In agreement with these results, an increase of expression of motility genes and a decrease in nodulation gene expression were found in the $f a d D$ mutant, suggesting a FadD-dependent regulation mechanism [2]. In a follow-up investigation carried out to determine differences in the lipidic composition between fadD mutants and wild type, we found that strains of $S$. meliloti and of E. coli lacking functional FadD accumulated significant amounts of free fatty acids upon entry to the stationary phase of growth. We showed that fatty acids, accumulated in the fadD mutant, were derived from complex membrane lipids without the occurrence of cell lysis. Furthermore, the expression analysis of cultures showed the upregulation of genes involved in fatty acid degradation in $S$. meliloti wild type with respect to its fadD mutant strain [3], indicating that fatty acids released from membrane lipids are degraded by $\beta$-oxidation in the stationary phase of growth. However, the accumulation of free fatty acids was not responsible for the swarming phenotype observed in a S. meliloti fadD-mutant. Instead, the fadD-associated swarming phenotype was due to increased formation of the volatile 2-tridecanone [4].

Whereas E. coli $\mathrm{K} 12$ contains a unique fadD gene, S. meliloti Rm1021 contains several Open Reading Frames (ORFs) with homology to long-chain fatty acyl-CoA ligases [2]. A deeper analysis of the $S$. meliloti Rm1021 genome identified that, besides FadD, a total of nine ORFs with homology to acyl-CoA synthetases are present (Table S1, Figure S1). Interestingly, each one of the two closest FadD homologues are located in different replicons of $S$. meliloti Rm1021. The chromosomally encoded FadD of S. meliloti (SMc02162) shows a 55\% identity to E. coli FadD, while its closest S. meliloti homologues SMb20650 (encoded in megaplasmid pSymB) and SMa0150 (encoded in megaplasmid pSymA), show an identity of about $30 \%$ to both the E. coli and the chromosome-encoded S. meliloti FadD (Table S1). Both SMb20650 and SMa0150 contain an AMP-binding motif as well as sequences that partially resemble the fatty acyl-CoA synthetase [FACS] signature motif common to all fatty acyl-CoA synthetases [2].

$\mathrm{SMb} 20650$ is a predicted long-chain fatty acyl-CoA ligase and its gene is cotranscribed with the gene coding for acyl carrier protein (ACP) SMb20651. Based on this, we hypothesized that SMb20650 could be involved in the acylation of SMb20651. The production of holo-SMb20651 in E. coli was achieved by co-expressing SMb20651 together with the phosphopantetheinyl transferase AcpS of S. meliloti. Additional expression of SMb20650 in the holo-SMb20651-forming E. coli strain, led to the in vivo formation of acylated SMb20651 [5]. SMa0150 shows a 75\% identity to Rhizobium leguminosarum bv trifolii malonyl-CoA synthetase (MatB), a 67\% identity to Bradyrhizobium japonicum MatB, and a 39\% identity to Streptomyces coelicolor MatB. The activity as malonyl-CoA synthetase has been demonstrated in vitro for the latter three enzymes [6-8]. Importantly, the conserved motif ERYGMTE found in prokaryotic as well as in mammalian and plant malonyl-CoA synthetases $[9,10]$ is present in SMa0150 (Figure S2). In S. meliloti, the gene coding for SMa0150 (MatB) is part of the operon matPQMAB that is induced by malonate, and a null mutant in mat $B$ is unable to grow on minimal medium containing malonate as the sole carbon source [11]. On the other hand, it was shown that the MatB of R. leguminosarum as well as the MatB of $S$. coelicolor have a broad substrate specificity [8,12].

In Pseudomonas aeruginosa, a total of six FadD homologues were functionally complementing an E. coli fadD mutant for its ability to grow in media containing fatty acids as the sole carbon source $[13,14]$. In the present study, we investigated whether the constitutive expression of either of the two closest homologues to S. meliloti FadD, SMb20650, or SMa0150, can complement the phenotypes exhibited by the fadD mutants of $S$. meliloti or E. coli. Given the pleiotropic phenotype of an S. meliloti fadD mutant and the suggested function of $S$. meliloti FadD as a regulator of expression [2], we also examined whether E. coli FadD reverts different phenotypes observed in a S. meliloti fadD mutant. Importantly, our studies reveal a crucial role of fatty acid degradation for survival in the stationary phase. 


\section{Materials and Methods}

\subsection{Bacterial Strains, Plasmids, and Growth Conditions}

Bacterial strains and plasmids used in this work are listed in Table 1. E. coli strains were grown at $30{ }^{\circ} \mathrm{C}$ either in Luria-Bertani (LB) broth or in M9 minimal medium [15]. Sinorhizobium meliloti strains were grown at $30^{\circ} \mathrm{C}$ either in complex tryptone yeast (TY) broth supplemented with $4.5 \mathrm{mM} \mathrm{CaCl}_{2}$ [16], in Robertsen minimal medium (MM) containing glutamate $(6.5 \mathrm{mM})$, mannitol $(55 \mathrm{mM})$, mineral salts (1.3 mM K $\mathrm{HPO}_{4}, 2.2 \mathrm{mM} \mathrm{KH} \mathrm{PO}_{4}, 0.6 \mathrm{mM} \mathrm{MgSO}_{4}, 0.34 \mathrm{mM} \mathrm{CaCl}, 22 \mu \mathrm{M} \mathrm{FeCl}, 0.86 \mathrm{mM} \mathrm{NaCl}$ ), vitamins (biotin $(0.2 \mathrm{mg} / \mathrm{L})$, and calcium pantothenate $(0.1 \mathrm{mg} / \mathrm{L}))$ [17], or in Sherwood MM with succinate $(8.3 \mathrm{mM})$, replacing mannitol as the carbon source [18]. To test the ability to use oleate as sole carbon source, $5 \mathrm{mM}$ oleate (Sigma) was used in defined media with the addition of $5 \mathrm{mg} / \mathrm{mL}$ Brij 58 and for the case of Robertsen $\mathrm{MM}, 2 \mathrm{mM} \mathrm{NH}_{4} \mathrm{Cl}$ was used as the nitrogen source instead of glutamate. Antibiotics were added, when required, to the following final concentrations $(\mu \mathrm{g} / \mathrm{mL})$ : carbenicillin, 100 and cloramphenicol, 20 for E. coli, or neomycin, 200 and tetracycline, 8 for S. meliloti. The mutation of the fadD gene in E. coli BL21 (DE3) was transduced from the fadD mutant JW1794-1 of the Keio Collection [19] by $\mathrm{P}_{\mathrm{vir}}$ transduction [20] selecting for kanamycin resistant colonies. Correct transfer of the fadD mutation in strain BfadD1 was corroborated by the absence of growth after plating on M9 MM agar plates containing $5 \mathrm{mM}$ sodium oleate as unique carbon source.

Table 1. Bacterial strains and plasmids used in this work.

\begin{tabular}{|c|c|c|}
\hline $\begin{array}{l}\text { Strain or } \\
\text { Plasmid }\end{array}$ & Relevant Characteristics ${ }^{a}$ & Reference or Source \\
\hline \multicolumn{3}{|l|}{ Escherichia coli } \\
\hline $\mathrm{DH} 5 \alpha$ & recA1, $\Phi 80$ lac $Z \Delta M 1 ;$ cloning strain & [21] \\
\hline S17-1 & $\begin{array}{l}\text { thi pro rec } A \text { hsdR } R^{-} \text {hsd } M^{+} \mathrm{RP} 4 \text { integrated in the } \\
\text { chromosome, 2-Tc::Mu, } \mathrm{Km}:: \mathrm{Tn} 7\left(\mathrm{Tp}^{\mathrm{R}} / \mathrm{Sm}^{\mathrm{R}}\right)\end{array}$ & [22] \\
\hline Y-Mel & Wild type strain & [23] \\
\hline YfadD1 & Y-Mel fadD::kan & [3] \\
\hline JW1794-1 & BW25113 fadD::kan & [19] \\
\hline BL21(DE3) & $\mathrm{F}^{-}$ompT hsdSB $\left(\mathrm{rB}^{-}, \mathrm{mB}^{-}\right)$gal dcm (DE3) & [24] \\
\hline BfadD1 & BL21(DE3) fadD::kan & This work \\
\hline \multicolumn{3}{|c|}{ Sinorhizobium meliloti } \\
\hline GR4 & Wild type strain & [25] \\
\hline QS77 & fadD::Tn5 insertion mutant derivative of GR4, $\mathrm{Nm}^{\mathrm{R}}$ & [2] \\
\hline \multicolumn{3}{|c|}{ J } \\
\hline pLysS & $\mathrm{Cm}^{\mathrm{R}}$; causes repression of $\mathrm{T} 7$ polymerase & [24] \\
\hline pET16b & Expression vector, $\mathrm{Cb}^{\mathrm{R}}$ & Novagen \\
\hline pET17b & Expression vector, $\mathrm{Cb}^{\mathrm{R}}$ & Novagen \\
\hline pAL55 & smb20650 in pBBR1MCS-5, Gm ${ }^{\mathrm{R}}$ & [5] \\
\hline pECH1 & sma0150 in pET16b, $\mathrm{Cb}^{\mathrm{R}}$ & This work \\
\hline pECH6 & smc02162 in $\mathrm{pET} 17 \mathrm{~b}, \mathrm{Cb}^{\mathrm{R}}$ & This work \\
\hline pECH7 & $s m b 20650$ in $\mathrm{pET} 17 \mathrm{~b}, \mathrm{Cb}^{\mathrm{R}}$ & This work \\
\hline $\mathrm{pECH} 8$ & E. coli fadD pET16b, $\mathrm{Cb}^{\mathrm{R}}$ & This work \\
\hline pBBR1MCS-3 & Broad-host range vector, $\mathrm{Tc}^{\mathrm{R}}$ & [26] \\
\hline pBBRD4 & $\begin{array}{l}\text { pBBR1MCS-3 derivative harbouring the fadD gene of } \\
\text { S. meliloti GR4, Tc }\end{array}$ & {$[2]$} \\
\hline pRK404 & Broad-host range vector, $\mathrm{Tc}^{\mathrm{R}}$ & [27] \\
\hline pRCanul1 & 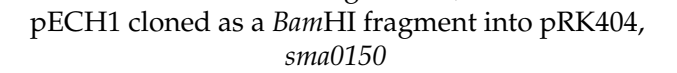 & This work \\
\hline pRCanul2 & $\begin{array}{l}\text { pECH6 cloned as a HindIII fragment into pRK404, } \\
\qquad s m c 02162\end{array}$ & This work \\
\hline pRCanul3 & $\begin{array}{l}\text { pECH7 cloned as a BglII fragment into pRK404, } \\
\qquad s m b 20650\end{array}$ & This work \\
\hline pRCanul4 & $\begin{array}{l}\text { pECH8 cloned as a BamHI fragment into pRK404, } \\
\qquad f a d D_{\text {Ecoli }}\end{array}$ & This work \\
\hline pNG28 & pET17b cloned in pRK404 & [28] \\
\hline
\end{tabular}

${ }^{\mathrm{a}} \mathrm{Tc}^{\mathrm{R}}, \mathrm{Tp}^{\mathrm{R}}, \mathrm{Sm}^{\mathrm{R}}, \mathrm{Km}^{\mathrm{R}}, \mathrm{Nm}^{\mathrm{R}}, \mathrm{Cm}^{\mathrm{R}}, \mathrm{Gm}^{\mathrm{R}}, \mathrm{Cb}^{\mathrm{R}}$ : tetracycline, trimethoprim, streptomycin, kanamycin, neomycin, cloramphenicol, gentamicin, and carbenicillin resistance, respectively. 


\subsection{DNA Manipulations}

Recombinant DNA techniques were carried out using standard procedures [15]. Restriction sites introduced with oligonucleotides primers are underlined. The gene sma0150 was amplified by PCR using specific primers (5'-ACCTTATCCATGGGCAACCATCTGTTCGACG-3' and $5^{\prime}$-AAAGGATCCCTACACACGCGCTTCGGCTC-3') and after digestion of the resulting fragment with $\mathrm{NcOI}$ and $\mathrm{Bam} \mathrm{HI}$ it was cloned into pET16b, yielding plasmid pECH1. The gene smc02162 was amplified by PCR using specific primers (5'-AGGAATCATATGGCGGAAGCAAGCACGC-3' and $5^{\prime}$-CCCAAGCTTCTATCCGCGCAGGTCCTTG-3') and after digestion of the resulting fragment with $\mathrm{NdeI}$ and HindIII it was cloned into pET17b, yielding plasmid pECH6. The E. colifadD gene was amplified by PCR using specific oligonucleotides (5'-AAATTCACCATGGTAACGGCATGTATATCATTTG- $3^{\prime}$ and $5^{\prime}$-ACAGGATCCTCAGGCTTTATTGTCCACTTTGC-3') and after digestion of the resulting fragment with $\mathrm{NcoI}$ and BamHI it was cloned into pET16b, yielding plasmid pECH8. Amplified DNA fragments were commercially sequenced by Eurofins Medigenomix (Martinsried, Germany) to confirm PCR fidelity. The gene $s m b 20650$ was obtained by NdeI/EcoRI digestion from pAL55 [5] and subcloned into $\mathrm{pET17b}$, yielding $\mathrm{pECH7}$. The $\mathrm{pET}$ constructions were linearized and cloned into pRK404 previously digested with BamHI or HindIII. The pRK404 derivatives were mobilized into S. meliloti QS77 by biparental mating using the E. coli S17-1 donor strain as previously described [22].

\subsection{In vivo Labeling of S. meliloti and E. coli with ${ }^{14} \mathrm{C}$-Acetate and Analysis of Lipid Extracts by Thin-Layer Chromatography (TLC)}

The lipid composition of the different S. meliloti and E. coli strains was determined following labelling with $\left[1-{ }^{14} \mathrm{C}\right]$-acetate as previously described [3]. Lipids from cell pellets were extracted according to the method of Bligh and Dyer [29] and lipids from spent media supernatants were extracted with equal volumes of acidified ethyl acetate $(0.1 \mathrm{~mL}$ glacial acetic acid per litre of ethyl acetate). Lipids obtained were analysed by one-dimensional thin-layer chromatography (TLC) using high-performance TLC silica gel 60 plates (Merck) and mobile-phase ethyl acetate-hexane-acetic acid $(60: 40: 5(v / v / v))$. Radioactivity was detected using a Storm 820 PhosphorImager (Amersham Biosciences). Image analysis and signal quantification were carried out using ImageQuant TL (Amersham Biosciences). E. coli BL21 (DE3)-derived strains were grown in M9 MM, and protein expression was induced by the addition of $0.1 \mathrm{mM}$ isopropyl- $\beta$-D-thiogalactopyranoside (IPTG) during the mid-exponential phase of bacterial growth $\left(\mathrm{OD}_{620 \mathrm{~nm}}=0.4\right)$. The cultures were collected $21 \mathrm{~h}$ after induction with IPTG. For labelling experiments, S. meliloti strains were grown on Robertsen MM. Cultures were labelled at $\mathrm{OD}_{620 \mathrm{~nm}}=0.1$ and collected after $66 \mathrm{~h}$ of growth. For each strain, labelling experiments were repeated 3 times and representative TLCs are shown.

\subsection{Surface Motility Assays}

The ability of S. meliloti strains to spread over surfaces was assayed essentially as previously described [30]. Briefly, S. meliloti was grown at $30{ }^{\circ} \mathrm{C}$ in TY broth to the late exponential phase. Cells were sedimented, washed twice in Robertsen $\mathrm{MM}$, and resuspended in 1/10 of the initial volume. Aliquots of $2 \mu \mathrm{L}$ of this cell suspension were dispensed onto the surface of plates with $20 \mathrm{~mL}$ of semisolid Robertsen MM containing 0.6\% Noble Agar Difco (BD) and allowed to dry for $10 \mathrm{~min}$. The plates were incubated at $30{ }^{\circ} \mathrm{C}$ for $24 \mathrm{~h}$. Pictures and measurements of the migration zones (determined as the colony diameter in millimetres) were taken two days later to allow for the accumulation of exopolysaccharides and a better visualization of the colonies.

\subsection{Cell Viable Counts of S. meliloti and E. coli}

Cell viability from liquid cultures of S. meliloti and E. coli strains was followed by determining colony forming units (CFU) at distinct time points [31]. Colonies growing on plates were counted after $18 \mathrm{~h}$ of incubation for E. coli or $48 \mathrm{~h}$ for S. meliloti. The volume of $S$. meliloti liquid cultures in the 
long-term cultivation experiment carried out in Robertsen MM was kept constant by adding sterile distilled water. The experiments were independently performed three times with three replicates for each dilution. The cell viability was expressed as $\mathrm{CFU} / \mathrm{mL}$ of culture.

\section{Results}

3.1. Growth of Sinorhizobium meliloti and Escherichia coli fadD Mutants on Oleate Cannot be Complemented by sma0150 or smb20650

As expected, mutation of the gene coding for long-chain fatty acyl-CoA synthetase in S. meliloti $(s m c 02162=f a d D)$ abolished the capacity to grow on oleate as sole carbon source [2]. Furthermore, this mutation leads to a swarming phenotype [2] and to the accumulation of endogenous free fatty acids [3]. In the present work, we investigated if all these phenotypes could be restored by heterologous complementation with E. coli fadD or by constitutive expression of sma0150 or smb20650, encoding the two closest FadD homologues of S. meliloti.

Previously, it was demonstrated that plasmid pBBRD4 carrying S. meliloti GR4 fadD under its own promoter was able to restore growth on oleate in an E. coli fadD mutant [2]. In this study, we have cloned the DNAs coding for ORFs smc02162 (fadD), sma0150 (matB), smb20650, and E. coli fadD in pET vectors (see Table 1) and afterwards, the different expression vectors have been recloned into the broad host range vector pRK404. As a control, a cointegration of vectors pET17b and pRK404 (pNG28) was used. Growth on oleate of strain QS77 can be complemented either by S. meliloti fadD or E. coli fadD, but constitutive expression of sma0150 or smb20650 could not complement for growth on oleate (Figure 1A). In order to be able to express pET vectors using the NOVAGEN expression system, we have created a fadD mutant of strain E. coli BL21(DE3). BfadD1 could not be complemented for growth on oleate by vectors expressing either sma0150 or smb20650, while it could be complemented by E. coli fadD or S. meliloti fadD (Figure 1B). These data suggest that SMa0150 or SMb20650 do not have the capacity to form oleoyl-CoA that would support the growth of E. coli or S. meliloti cells deficient in long-chain acyl-CoA synthetase. 

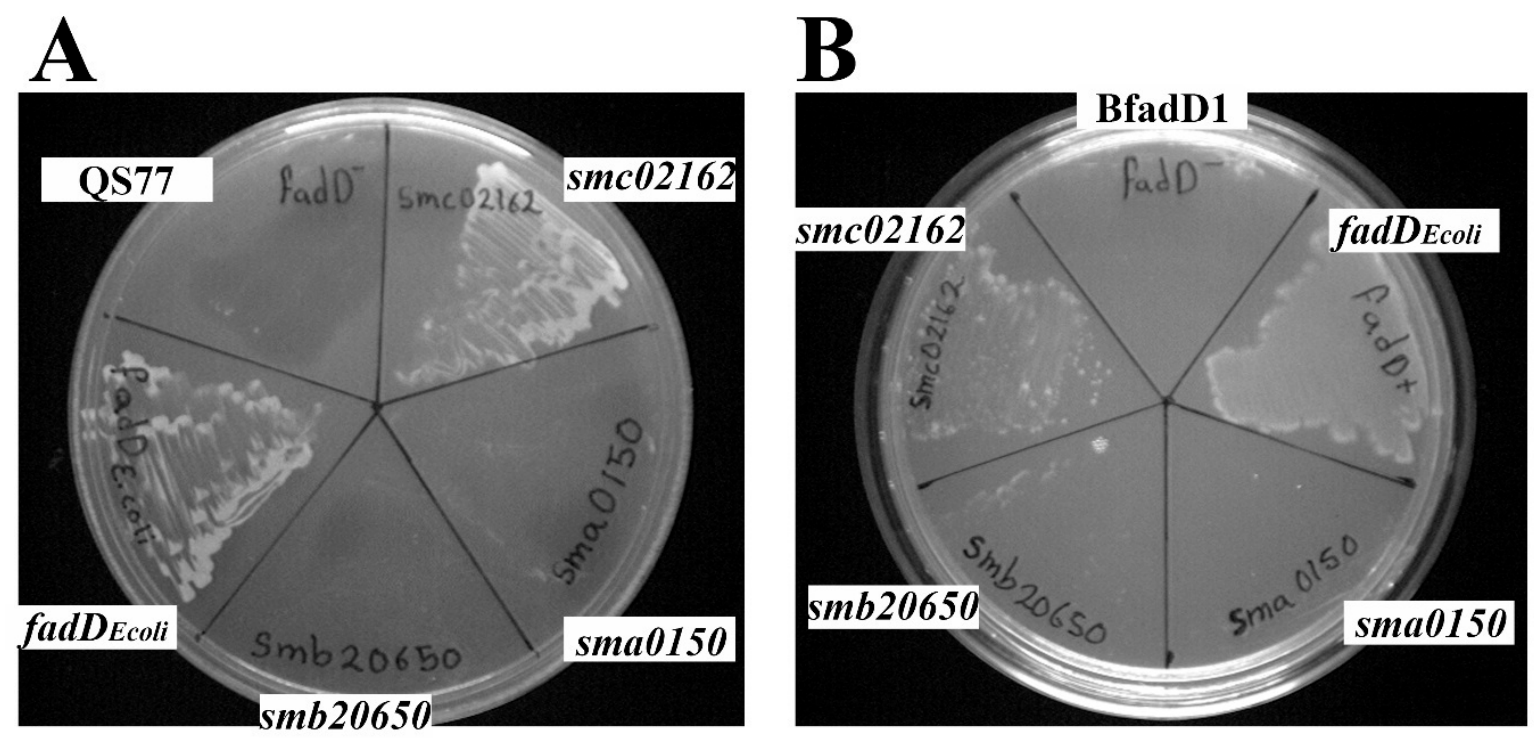

Figure 1. Expression of $s m b 20650$ or sma0150 in S. meliloti or E. coli fadD mutants does not restore growth on oleate. (A) The growth of S. meliloti fadD mutant QS77 carrying the empty vector pNG28 (QS77), pRCanul2 (smc02162), pRCanul1 (sma0150), pRCanul3 (smb20650), or pRCanul4 (fadD Ecoli) on Robertsen minimal medium (MM) with $5 \mathrm{mM}$ oleate as a sole carbon source and $5 \mathrm{mg} / \mathrm{mL}$ Brij 58 . (B) The growth of E. coli fadD mutant BfadD1 pLysS carrying pET17b (BfadD1), pECH8 (fadD Ecoli), pECH1 (sma0150), pECH7 (smb20650), or pECH6 (smc02162) on M9 MM containing 5 mM oleate as a sole carbon source, $5 \mathrm{mg} / \mathrm{mL}$ Brij 58 , and $0.1 \mathrm{mM}$ isopropyl- $\beta$-D-thiogalactopyranoside (IPTG). $S$. meliloti QS77 pNG28 and E. coli BfadD1 pLysS pET17b were used as controls.

\subsection{Free Fatty Acid Accumulation in Sinorhizobium meliloti and Escherichia coli fadD Mutants Cannot be} Reverted by sma0150 or smb20650

We found that mutants in $f a d D$ of $S$. meliloti and E. coli accumulated free fatty acids in the stationary phase [3]. Such fatty acid accumulation does not occur in QS77 carrying the fadD-bearing plasmid pBBRD4 [3]. Thin-layer chromatography (TLC) analyses of lipid extracts from cells demonstrate that wild type $S$. meliloti GR4 cells carrying the empty vector pNG28 do not accumulate free fatty acids while its fadD mutant QS77 carrying pNG28 shows fatty acid accumulation (Figure 2A, lanes 1 and 2). The expression of $s m c 02162$ ( $f a d D)$ from pRCanul2 or of E. coli fadD from pRCanul4 suppresses fatty acid accumulation in the fadD mutant (Figure 2A, lanes 3 and 6). However, the expression of the two closest S. meliloti fadD homologs sma0150 or smb20650 into the fadD mutant QS77 does not suppress the fatty acid accumulation phenotype (Figure 2A, lanes 4 and 5). Similar effects were observed in culture supernatants although only a modest accumulation of fatty acids occurred (Figure 2B). The quantification of spots showed that labelled free fatty acids accumulated in supernatants were between $10 \%$ and $16 \%$ of the amount of free fatty acids accumulated in cells (Table S2 and Figure S3). Lipid extracts of cells and spent mediaof $S$. meliloti strains lacking smc02162 or E. coli fadD accumulated other unidentified hydrophobic compounds in addition to free fatty acids (Figure 2).

In a previous work, we have shown that a fadD mutant of E. coli strain Y-Mel accumulated free fatty acids. As for the case of strain Y-Mel [3], a fadD mutant of E. coli BL21(DE3) was accumulating significant amounts of free fatty acids (Figure 3A, lanes 2). The expression of E. coli fadD or S. meliloti fadD (smc02162) eliminated fatty acid accumulation (Figure 3A, lanes 3 and 6) but the expression of sma0150 or smb20650 in the E. coli mutant background did not eliminate free fatty acid accumulation (Figure 3A, lanes 4 and 5). In the spent media of E. coli BfadD1 derivatives that lack functional long-chain fatty acid-CoA ligase, a higher amount of free fatty acids to those found in cell extracts is observed (Figure 3B, Figure S4 and Table S3). The amount of labelled free fatty acids associated with cells were about $50 \%$ of the free fatty acids observed in supernatants (Table S3 and Figure S4). The bigger proportion of free fatty acids in the supernatants of E. coli fadD mutants might be due to 
the fact that E. coli cultures were in the stationary phase for more generation times than $S$. meliloti cultures. In the conditions studied, the generation time for E. coli was $2 \mathrm{~h}$ and for S. meliloti it was 8 h. Interestingly, the supernatants of all E. coli cultures present a hydrophobic compound with an $R_{\mathrm{f}}$ value similar to that of the unidentified compound observed in supernatants of $S$. meliloti lacking a functional fadD (Figure 2B, lanes 2, 4 and 5). However, in E. coli its presence is not correlated with the absence of FadD activity (Figure 3B).

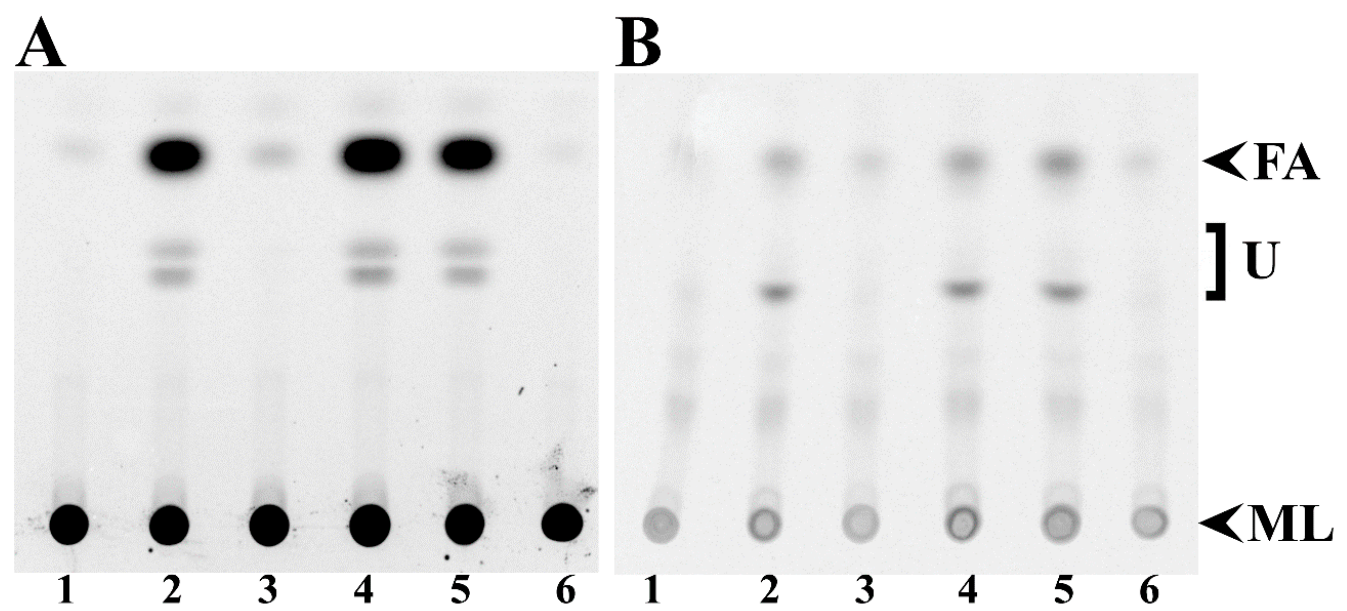

Figure 2. Expression of sma0150 or smb20650 does not abolish free fatty acid accumulation in fadD mutants of S. meliloti. Thin-Layer Chromatography (TLC) analyses of cellular lipid extracts (A) and lipid extracts of spent media (B) obtained either from wild type S. meliloti GR4 carrying the empty vector pNG28 (lane 1) or from its fadD mutant QS77 carrying either pNG28 (lane 2), pRCanul2 (smc02162, lane 3), pRCanul1 (sma0150, lane 4), pRCanul3 (smb20650, lane 5), or pRCanul 4 (fadD Ecoli, lane 6) grown on Robertsen $\mathrm{MM}$ into the stationary phase $\left(\mathrm{OD}_{620}=1.2,66 \mathrm{~h}\right.$ of growth). The membrane lipids (ML) did not migrate from the origin and the spot for fatty acids (FA) is indicated. U: unidentified lipid spots. A single experiment representative of three repetitions is shown.

A

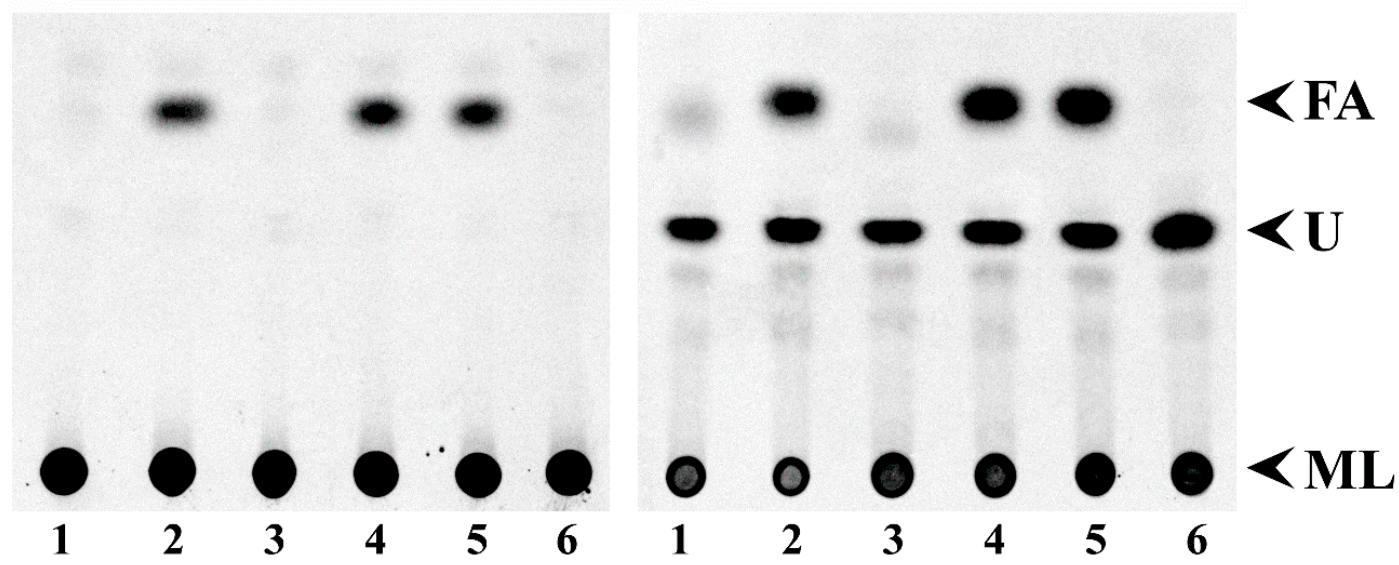

Figure 3. Expression of sma0150 or smb20650 does not abolish free fatty acid accumulation in fadD mutants of E. coli. TLC analyses of cellular lipid extracts (A) and lipid extracts of spent media (B) obtained either from wild type E. coli BL21 (DE3) pLysS carrying the empty vector pET17b (lane 1) or from its fadD mutant BfadD1 pLysS carrying pET17b (lane 2), pECH8 (fadD Ecoli, lane 3), pECH1 (sma0150, lane 4), pECH7 (smb20650, lane 5), or pECH6 (smc02162, lane 6), grown on M9 MM for $26 \mathrm{~h}$. The membrane lipids (ML) did not migrate from the origin, and the spot for fatty acids (FA) is indicated. U: unidentified lipid spot. A single experiment representative of three repetitions is shown. 
3.3. Effect of the E. coli fadD, S. meliloti fadD (smc02162), and S. meliloti Genes sma0150 and smb20650 on the Surface Motility of a fadD Mutant of S. meliloti (QS77)

It has been shown that loss of function of smc02162 ( $\mathrm{fadD})$ promotes surface motility in S. meliloti [2,32]. This phenotype is reverted to the wild type behaviour by introducing smc02162 in trans in a pBBR1MCS-3 derivative construct [2]. To test if this effect is exerted exclusively by the $S$. meliloti fadD gene or by any other fatty acyl-CoA ligase, the motility behaviours of fadD mutant (QS77) derivatives expressing the E. coli fadD gene, or either genes coding for the two closest $S$. meliloti FadD homologues, sma0150 (matB) and smb20650, were assayed on semisolid Robertsen MM. As shown in Figure 4, only plasmids pRCanul2 and pRCanul4, containing smc02162 and E. coli fadD, respectively, were able to inhibit surface translocation of the mutant to levels similar to those exhibited by the wild type strain (Figure 4B and Figure S5). These results indicate that the regulation of surface motility is specific to the long-chain fatty acyl-CoA ligase FadD.
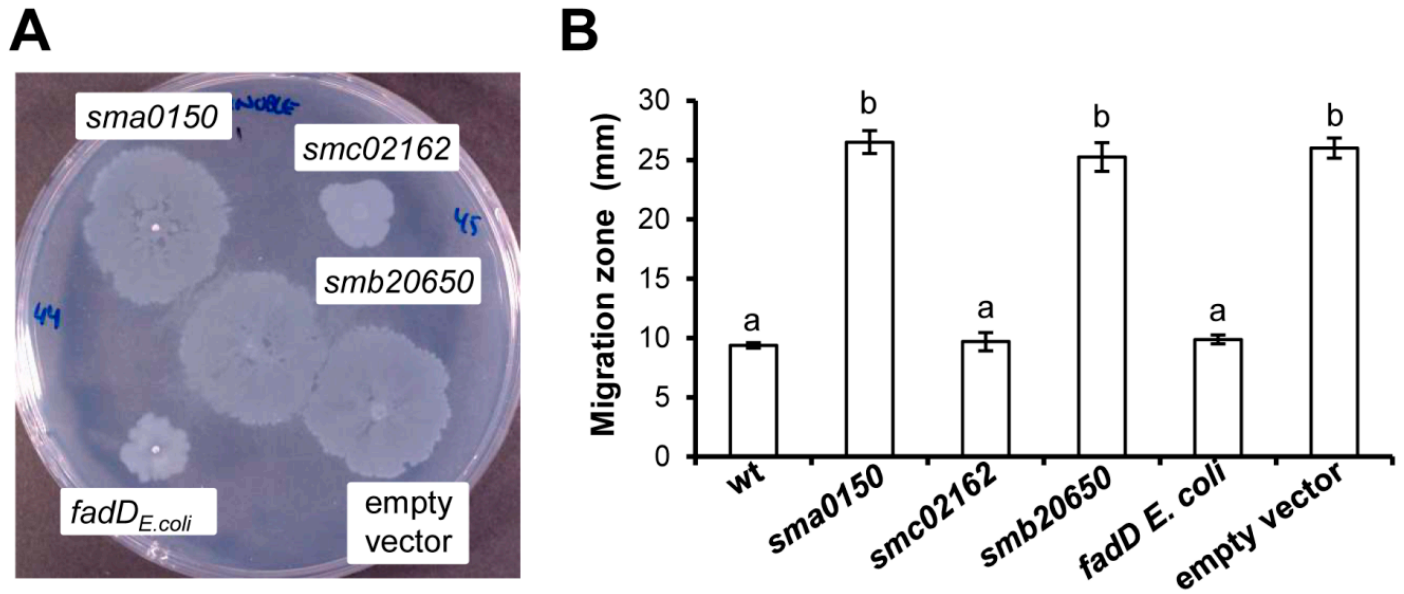

Figure 4. Effect of different fadD homologues on the surface motility of S. meliloti QS77 (smc02162-). (A) A representative picture of surface motility on Robertsen semisolid MM (0.6\% Noble agar) shown by QS77 harbouring either pRCanul1 (sma0150), pRCanul2 (smc02162), pRCanul3 (smb20650), pNG28 (empty vector), or pRCanul 4 ( $\left.f a d D_{E c o l i}\right)$. (B) The surface expansion shown by the wild type strain and QS77 derivatives shown in (A). The bars and error bars represent the mean and standard error of the migration zones obtained for each strain from two independent biological experiments with at least four technical replicates. Different letters indicate significant differences according to an analysis-of-variance test $(p \leq 0.05)$.

\subsection{Absence of fadD Reduces Survival Rates in the Stationary Phase of Growth}

Since strains lacking a functional fadD cannot reutilize free fatty acids released from membranes as a carbon source, we speculated that the wild type should have a metabolic advantage over the mutant in the stationary phase [3]. In a first experiment with the strains Y-Mel and its fadD mutant YfadD, viable counts of the mutant strain after $52 \mathrm{~h}$ of growth were reduced to $33 \%$ with respect to the wild type strain (Figure 5A). Next, we checked for survival rates of strain BL21 (DE3) pLysS and its fadD mutant BfadD1 carrying either an empty plasmid or the expression plasmids for E. coli fadD, S. meliloti fadD (smc02162), or S. meliloti genes sma0150 or smb20650. Although, to a lesser extent, again, a significant difference in colony forming units was observed between the wild type and its fadD mutant with a reduction of the survival to $75 \%$ at $52 \mathrm{~h}$ and to $62 \%$ at $72 \mathrm{~h}$. Viable counts were similar to the wild type in strains complemented either with E. coli or S. meliloti fadD, while survival rates could not be restored by sma0150 or smb20650 (Figure 5B), probably due to the inability to consume free fatty acids. 

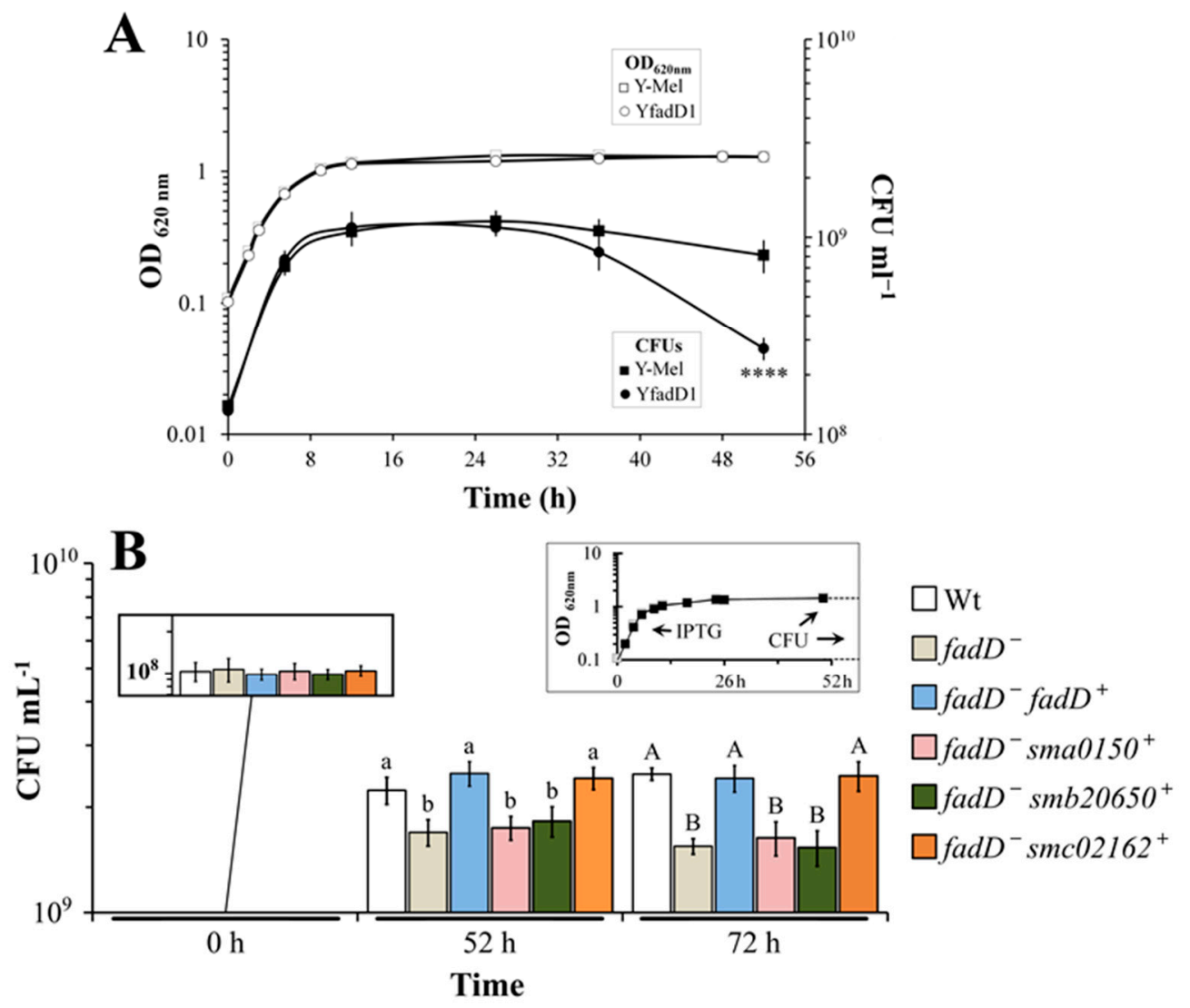

Figure 5. Absence of $f a d D$ reduces survival in the stationary phase of E. coli. (A) The growth curves and number of viable cells (CFU) of E. coli Y-Mel and its fadD mutant YfadD1 grown in M9 MM. Open symbols represent optical density (OD) whereas $\mathrm{CFU} \mathrm{ml} \mathrm{m}^{-1}$ are represented with filled symbols. The statistical significance was calculated in Prism 8.4 using an unpaired two-tailed $t$-test in which the fadD mutant was compared to the parental strain. The statistical significance is shown $(* * * *<0.0001)$. (B) The number of viable cells of E. coli wild type BL21 (DE3) pLysS carrying the empty vector pET17b (Wt) or from its fadD mutant BfadD1 pLysS carrying pET17b (fadD $\left.{ }^{-}\right), \mathrm{pECH8}\left(\right.$ fadD $\left.^{+}\right), \mathrm{pECH} 1$ (sma0150), pECH7 (smb20650), or pECH6 (smc02162) after 0, 52, and $72 \mathrm{~h}$ of growth in M9 MM. The inset in the upper right corner represents the OD of the cultures, and the arrows point to the addition of IPTG and to $52 \mathrm{~h}$ when $\mathrm{CFU}$ were determined. From $52 \mathrm{~h}$ to $72 \mathrm{~h}$ the OD of the different cultures maintained constant. For each strain three independent cultures were analysed. The error bars represent the SD. Different letters for bars at 52 and $72 \mathrm{~h}$ indicate significant differences according to an analysis-of-variance test $(p<0.001)$.

In order to test if the absence of $f a d D$ also reduces viability in S. meliloti, we followed the optical density (OD) and the number of viable cells of cultures of S. meliloti GR4 and its fadD mutant QS77 in a long-term cultivation experiment (see material and methods). Even after 22 days of growth, the OD of the cultures remained constant and there was no difference between wild type and mutant (Figure S6). However, the number of viable cells of both cultures started to decrease after 7 days, and, after 9 days, a significant difference was observed between them. From day 9 to day 22, the number of colony forming units (CFUs) obtained from mutant cultures was decreased from 45\% (day 14) to 30\% (day 22) compared to those obtained from the wild type (Figure S6). When Sherwood MM [18] was used instead of Robertsen MM [17], a significant decrease in viability was already observed after 2 days of growth (Figure 6). This difference in survival might be due to the significantly lower concentration of carbon source present in Sherwood MM of $8.3 \mathrm{mM}$ succinate versus $55 \mathrm{mM}$ of mannitol present in Robertsen MM. The viability of wild type and fadD mutant was decreasing and the numbers of viable cells recuperated from the fadD mutant were less than $50 \%$ of those recovered from the wild 
type (Figure 6). Importantly, the fadD mutant carrying a plasmid harboring fadD shows a survival rate similar to the wild type, while the mutant with the empty vector is surviving to a lesser extent (Figure 6). Therefore, the presence of FadD confers an increased survival to cultures of E. coli and S. meliloti in the stationary phase.

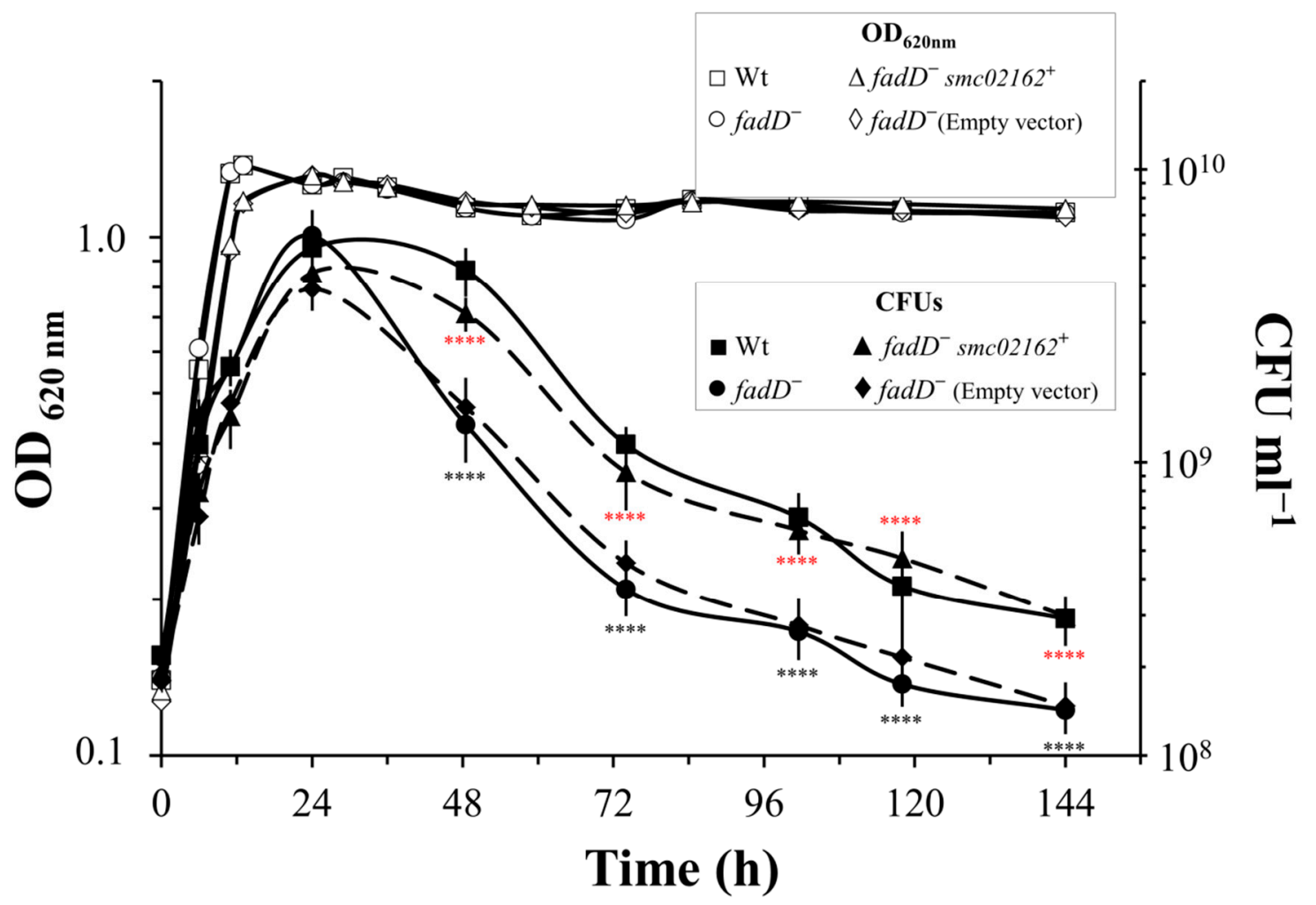

Figure 6. Expression of $f a d D$ in a $S$. meliloti fadD mutant recovers survival rate. The growth curves and viable cells (CFU) of wild type S. meliloti GR4, its fadD mutant QS77, QS77 harbouring pRCanul2 (smc02162), and QS77 harbouring empty plasmid pNG28 grown on Sherwood MM. Open symbols represent optical density, whereas CFU are represented with filled symbols. The statistical significance was calculated in Prism 8.4 using an unpaired two-tailed $t$-test in which the fadD mutant QS77 was compared to the parental strain GR4 (black asterisks), and the QS77 harbouring an empty plasmid was compared to the QS77 harbouring smc02162 (red asterisks). The statistical significance is shown $\left({ }^{* * * *} p<0.0001\right)$. For each strain, three independent cultures were analysed. The error bars represent the SD.

\section{Discussion}

A mutant in the fadD of $S$. meliloti is unable to grow on oleate as the sole carbon source, shows increased surface motility with respect to the wild type, and accumulates free fatty acids in the stationary phase [2,3]. In this work, we have shown that all of these different phenotypes can be complemented by the expression of the gene encoding the E. coli FadD homologue, indicating that there are no functional differences between the $S$. meliloti and the E. coli FadD proteins. However, none of these phenotypes could be reverted by any of the closest S. meliloti FadD homologues, SMa0150 or

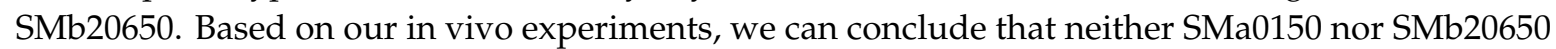
have the capacity of linking long-chain fatty acids efficiently to CoA.

The fadD mutants of $S$. meliloti accumulate free fatty acids at the entry of the stationary phase and the source for them are mainly membrane phospholipids [3]. However, little is known about the activities that release fatty acids. Recently, we have identified in S. meliloti a diacylglycerol lipase that is, in part, responsible for the release of fatty acids [33]. In the absence of FadD, free fatty acids are accumulated in the stationary phase, while in wild type strains the fatty acids are 
consumed by $\beta$-oxidation [3]. We hypothesized that this extra carbon source available for the wild type might confer a survival advantage in comparison with their counterparts that lack FadD activity and are therefore unable to utilize the accumulated fatty acids. Indeed, inactivation of $f a d D$ in $E$. coli Y-Mel reduces its survival growth to $33 \%$ with respect to the wild type strain (Figure 5A). In similar conditions of growth, a fadD mutant of E. coli BL21(DE3) lost $25 \%$ of its viability with respect to the wild type (Figure 5B). Importantly, cell viability is restored to wild type levels when such a mutant is complemented with $S$. meliloti or E. coli fadD but not after the expression of sma0150 or smb20650 (Figure 5B). Different investigations have made use of E. coli fadD mutants. Fulda et al. [34] cloned and sequenced the E. coli K12 fadD gene by complementing the fadD phenotype with different deletion clones. Moreover, an E. coli BL21(DE3) derivative mutated in fadD was used to test for complementation of growth on oleate with five different long-chain acyl-CoA synthetases from rats. Only one of them could complement for growth on oleate [35]. Complementation of an E. coli fadD mutant by the expression of $f a d D$ from $E$. coli restored its ability to grow on $\mathrm{C} 12$, as well as growth on the non-inducing fatty acids of $\beta$-oxidation $\mathrm{C} 10$ and $\mathrm{C} 8$. These results show that FadD dosage plays an important role in the regulation of $\beta$-oxidation [36].

Most bacteria reduce their size considerably upon entry into the stationary phase as a result of reductive division and dwarfing. Degradation of the membrane components is part of the dwarfing process of non-differentiating bacteria under starvation for exogenous carbon and energy generating small, coccoid cells (reviewed in [37]). Farewell et al. [38] found in E. coli an increased expression of genes of the FadR regulon during the entry of cells into the stationary phase and mutants unable to increase their expression survive long-term stasis poorly. These authors suggested that the Fad regulon, apart from being required for growth on exogenous long-chain fatty acids, might be involved in providing the growth arrested cells with endogenous carbon and energy during dwarfing [38]. Comparing the cells of $S$. meliloti $\mathrm{Rm} 1021$ at the entry of the stationary phase with cells in the exponential phase of growth, Sauviac et al. [39] found a significant up-regulation of the operon smc02229-fadAB that is required for fatty acid $\beta$-oxidation. The $\mathrm{M}$ values of this microarray study for the comparison of stationary phase versus exponential phase of growth for the genes smc02229 (fadE), fadA, and fadB were 3, 2.7, and 2.8, respectively [40]. We compared the global expression of cultures of S. meliloti Rm1021 at the entry of the stationary phase for the wild type strain and its fadD mutant and found strong up-regulation of fatty acid degradation genes in the wild type strain [3]. The fadD mutant accumulates about $100 \mathrm{nmol}$ of free fatty acids associated to the cells per $\mathrm{ml}$ of culture, while the wild type contains less than $1 \mathrm{nmol} / \mathrm{mL}$ culture. Given the strong induction of fatty acid degradation genes, we suggested that free fatty acids were consumed in the wild type and accumulated in the fadD mutant. Furthermore, the fadD mutants of E. coli accumulated a significant amount of free fatty acids both in the cell-associated fraction and in the culture supernatant ([3] and Figure 3). We propose that the higher survival in the stationary phase observed for the wild type strains of S. meliloti and of E. coli with respect to their fadD mutants (Figures 5 and 6 ) is due to the capacity of the wild type to metabolize endogenous fatty acids.

The $\beta$-oxidation of fatty acids has usually been studied as a property to utilize exogenous long-chain fatty acids [1]. However, our present and previous results emphasize the important role of the fatty acid degradation system in the utilization of endogenous fatty acids. The capacity to use endogenous fatty acids provides the cells with extra carbon source during starvation, which results in a better rate of survival in the stationary phase. In E. coli, an alternative complete $\beta$-oxidation system has been described that works under anaerobic conditions in the presence of nitrate or fumarate as terminal electron acceptors [41]. ORFs coding for this pathway are found in the genomes of E. coli, Salmonella, Klebsiella, Yersinia, and Vibrio. It is likely that the anaerobic $\beta$-oxidation system gives them an important extra carbon source under starving conditions.

The biochemical function of long-chain fatty acyl-CoA synthetase is necessary for activation of fatty acids, thereby preparing them for subsequent degradation by $\beta$-oxidation. However, this enzyme activity also affects a number of different phenotypes. Several studies suggest a role of 
long-chain fatty acyl-CoA synthetase in pathogenesis since the inactivation of $f a d D$ affects virulence or colonization in Pseudomonas aeruginosa [13], Salmonella enterica, serovar Typhimurium [42], and Neisseria meningitidis [43]. The expression of fadD is involved in antibiotic production in Streptomyces coelicolor [44]. Furthermore, a lack of long-chain fatty acyl-CoA synthetase in the fungal pathogen Candida albicans led to a significant reduction in metabolic activity during biofilm formation [45], while the $\beta$-oxidation of fatty acids is required for fruiting body development in Myxococcus xanthus [46].

\section{Conclusions}

The capacity of fatty acid utilization from the extracellular environment has been described for many Gram-negative as well as for Gram-positive bacteria [43,47] and for yeast [45,48]. It is likely that all microorganisms, pathogenic or environmental, with the capacity to degrade fatty acids benefit from the utilization of exogenous as well as endogenous fatty acids. Our data reinforce the importance of a functional system for fatty acid degradation in providing a better survival rate in the stationary phase.

Supplementary Materials: The following are available online at http://www.mdpi.com/2076-2607/8/4/470/s1: Figure S1: Multiple sequence alignments of S. meliloti Rm1021 FadD (SMc02162) with E. coli FadD (FadDEc) and different ORFs of S. meliloti Rm1021 with homology to SMc02162; Figure S2: Multiple sequence alignments of $\mathrm{SMa} 0150$ with characterized malonyl-CoA synthetases; Figure S3: Graphical representation of spot intensities of free fatty acids formed in different S. meliloti strains; Figure S4: Graphical representation of spot intensities of free fatty acids formed in different $E$. coli strains; Figure S5: Effect of different fadD homologues on the surface motility of S. meliloti QS77 (smc02162-); Figure S6: Absence of fadD reduces survival in the stationary phase of S. meliloti; Table S1: Results of the pairwise sequence alignments of SMc02162 (S. meliloti FadD) with E. coli FadD or with different acyl-CoA synthetases of the S. meliloti Rm1021 genome; Table S2: Quantification of free fatty acids in different S. meliloti strains; Table S3: Quantification of free fatty acids in different E. coli strains.

Author Contributions: Conceptualization, M.J.S. and I.M.L.-L.; validation, all authors; formal analysis, Á.d.1.C.P.-C., M.J.S. and I.M.L.-L.; investigation, Á.d.l.C.P.-C.; G.R.-H. and J.N.; writing-original draft preparation, Á.d.l.C.P.-C.; writing—review and editing, all authors; visualization, Á.d.l.C.P.-C., G.R.-H., J.N., M.J.S. and I.M.L.-L.; supervision, M.J.S., O.G. and I.M.L.-L.; project administration, M.J.S. and I.M.L.-L; funding acquisition, M.J.S.; O.G. and I.M.L.-L. All authors have read and agreed to the published version of the manuscript.

Funding: This research was funded by grants from CONACyT/Mexico (153998 and 253549), from the Spanish Ministry /MINECO/MCIU/AEI (BIO2013-42801-P and PGC2018-096477-B-I00) and FEDER funds (EU). The APC was funded by Universidad Nacional Autónoma de México. G.R-H was supported by a postdoctoral fellowship from the DGAPA (Dirección General de Asuntos del Personal Académico)-UNAM (Universidad Nacional Autónoma de México). Á.d.l.C.P.-C. was supported by CONACyT through the “Cátedras CONACyT para Jóvenes Investigadores" Programme (Project \#609).

Acknowledgments: We thank Angeles Moreno (Centro de Ciencias Genómicas) for excellent technical assistance.

Conflicts of Interest: The authors declare no conflict of interest.

\section{References}

1. Rock, C.O. Fatty acid and phospholipid metabolism in prokaryotes. In Biochemistry of Lipids, Lipoproteins and Membranes, 5th ed.; Vance, D.E., Vance, J.E., Eds.; Elsevier: San Diego, CA, USA, 2008; pp. 59-96. [CrossRef]

2. Soto, M.J.; Fernández-Pascual, M.; Sanjuan, J.; Olivares, J. A fadD mutant of Sinorhizobium meliloti shows multicellular swarming migration and is impaired in nodulation efficiency on alfalfa roots. Mol. Microbiol. 2002, 43, 371-382. [CrossRef] [PubMed]

3. Pech-Canul, Á.; Nogales, J.; Miranda-Molina, A.; Álvarez, L.; Geiger, O.; Soto, M.J.; López-Lara, I.M. FadD is required for utilization of endogenous fatty acids released from membrane lipids. J. Bacteriol. 2011, 193, 6295-6304. [CrossRef] [PubMed]

4. López-Lara, I.M.; Nogales, J.; Pech-Canul, Á.; Calatrava-Morales, N.; Bernabéu-Roda, L.M.; Durán, P.; Cuéllar, V.; Olivares, J.; Alvarez, L.; Palenzuela-Bretones, D.; et al. 2-Tridecanone impacts surface-associated bacterial behaviours and hinders plant-bacteria interactions. Environ. Microbiol. 2018, 20, $2049-2065$. [CrossRef] [PubMed]

5. Ramos-Vega, A.L.; Dávila-Martínez, Y.; Sohlenkamp, C.; Contreras-Martínez, S.; Encarnación, S.; Geiger, O.; López-Lara, I.M. SMb20651 is another acyl carrier protein from Sinorhizobium meliloti. Microbiology 2009, 155, 257-267. [CrossRef] [PubMed] 
6. An, J.H.; Lee, G.Y.; Jung, J.-W.; Lee, W.; Kim, Y.S. Identification of residues essential for a two-step reaction by malonyl-CoA synthetase from Rhizobium trifolii. Biochem. J. 1999, 344, 159-166. [CrossRef]

7. Koo, H.M.; Kim, Y.S. Identification of active-site residues in Bradyrhizobium japonicum malonyl-Coenzyme A synthetase. Arch. Biochem. Biophys. 2000, 378, 167-174. [CrossRef]

8. Hughes, A.J.; Keatinge-Clay, A. Enzymatic extender unit generation for in vitro polyketide synthase reactions: Structural and functional showcasing of Streptomyces coelicolor MatB. Chem. Biol. 2011, 18, 165-176. [CrossRef]

9. Witkowski, A.; Thweatt, J.; Smith, S. Mammalian ACSF3 protein is a malonyl-CoA synthetase that supplies the chain extender units for mitochondrial fatty acid synthesis. J. Biol. Chem. 2011, 286, 33729-33736. [CrossRef]

10. Chen, H.; Kim, H.U.; Weng, H.; Browse, J. Malonyl-CoA synthetase, encoded by Acyl Activating Enzyme13, is essential for growth and development of Arabidopsis. Plant Cell 2011, 23, 2247-2262. [CrossRef]

11. Chen, A.-M.; Wang, Y.-B.; Jie, S.; Yu, A.-Y.; Luo, L.; Yu, G.-Q.; Zhu, J.-B.; Wang, Y.-Z. Identification of a TRAP transporter for malonate transport and its expression regulated by GtrA from Sinorhizobium meliloti. Res. Microbiol. 2010, 161, 556-564. [CrossRef]

12. Pohl, N.L.; Hans, M.; Lee, H.Y.; Kim, Y.S.; Cane, D.E.; Khosla, C. Remarkably broad substrate tolerance of malonyl-CoA synthetase, an enzyme capable of intracellular synthesis of polyketide precursors. J. Am. Chem. Soc. 2001, 123, 5822-5823. [CrossRef] [PubMed]

13. Kang, Y.; Zarzycki-Siek, J.; Walton, C.B.; Norris, M.H.; Hoang, T.T. Multiple FadD acyl-CoA synthetases contribute to differential fatty acid degradation and virulence in Pseudomonas aeruginosa. PLoS ONE 2010, 5, e13557. [CrossRef]

14. Zarzycki-Siek, J.; Norris, M.H.; Kang, Y.; Sun, Z.; Bluhm, A.P.; McMillan, I.A.; Hoang, T.T. Elucidating the Pseudomonas aeruginosa fatty acid degradation pathway: Identification of additional fatty acyl-CoA synthetase homologues. PLoS ONE 2013, 8, e64554. [CrossRef] [PubMed]

15. Sambrook, J.F.; Russell, D. Molecular Cloning: A Laboratory Manual (3-Volume Set), 3rd ed.; Cold Spring Harbor Laboratory: Cold Spring Harbor, NY, USA, 2001; Volume 1, p. 2100.

16. Beringer, J.E. R Factor Transfer in Rhizobium leguminosarum. Microbiology 1974, 84, 188-198. [CrossRef] [PubMed]

17. Robertsen, B.K.; Åman, P.; Darvill, A.G.; McNeil, M.; Albersheim, P. Host-symbiont interactions. V. The structure of acidic extracellular polysaccharides secreted by Rhizobium leguminosarum and Rhizobium trifolii. Plant Physiol. 1981, 67, 389-400. [CrossRef] [PubMed]

18. Sherwood, M.T. Improved synthetic medium for the growth of Rhizobium. J. Appl. Bacteriol. 1970, 33, 708-713. [CrossRef]

19. Baba, T.; Ara, T.; Hasegawa, M.; Takai, Y.; Okumura, Y.; Baba, M.; Datsenko, K.A.; Tomita, M.; Wanner, B.L.; Mori, H. Construction of Escherichia coli K-12 in-frame, single-gene knockout mutants: The Keio collection. Mol. Syst. Biol. 2006, 2, 2006.0008. [CrossRef]

20. Thomason, L.C.; Costantino, N.; Court, D.L. E. coli genome manipulation by P1 transduction. Curr. Protoc. Mol. Biol. 2007, 79, 11-18. [CrossRef]

21. Hanahan, D. Studies on transformation of Escherichia coli with plasmids. J. Mol. Biol. 1983, 166, 557-580. [CrossRef]

22. Simon, R.; Priefer, U.; Pühler, A. A broad host range mobilization system for in vivo genetic engineering: Transposon mutagenesis in Gram negative bacteria. Nat. Biotechnol. 1983, 1, 784-791. [CrossRef]

23. Rickenberg, H.V.; Lester, G. The preferential synthesis of $\beta$-galactosidase in Escherichia coli. Microbiology 1955, 13, 279-284. [CrossRef] [PubMed]

24. Studier, F.W. Use of bacteriophage T7 lysozyme to improve an inducible T7 expression system. J. Mol. Biol. 1991, 219, 37-44. [CrossRef]

25. Casadesús, J.; Olivares, J. Rough and fine linkage mapping of the Rhizobium meliloti chromosome. Mol. Gen. Genet. 1979, 174, 203-209. [CrossRef] [PubMed]

26. Kovach, M.E.; Elzer, P.H.; Hill, D.S.; Robertson, G.T.; Farris, M.A.; Roop, R.M.; Peterson, K.M. Four new derivatives of the broad-host-range cloning vector pBBR1MCS, carrying different antibiotic-resistance cassettes. Gene 1995, 166, 175-176. [CrossRef]

27. Ditta, G.; Schmidhauser, T.; Yakobson, E.; Lu, P.; Liang, X.-W.; Finlay, D.R.; Guiney, D.; Helinski, D.R. Plasmids related to the broad host range vector, pRK290, useful for gene cloning and for monitoring gene expression. Plasmid 1985, 13, 149-153. [CrossRef]

28. González-Silva, N.; López-Lara, I.M.; Reyes-Lamothe, R.; Taylor, A.M.; Sumpton, D.; Thomas-Oates, J.; Geiger, O. The dioxygenase-encoding ols $\mathrm{D}$ gene from Burkholderia cenocepacia causes the hydroxylation of the amide-linked fatty acyl moiety of ornithine-containing membrane lipids. Biochemistry 2011, 50, 6396-6408. [CrossRef] 
29. Bligh, E.G.; Dyer, W.J. A rapid method of total lipid extraction and purification. Can. J. Biochem. Phys. 1959, 37, 911-917. [CrossRef]

30. Bernabéu-Roda, L.; Calatrava-Morales, N.; Cuéllar, V.; Soto, M.J. Characterization of surface motility in Sinorhizobium meliloti: Regulation and role in symbiosis. Symbiosis 2015, 67, 79-90. [CrossRef]

31. Hoben, H.J.; Somasegaran, P. Comparison of the pour, spread, and drop plate methods for enumeration of Rhizobium spp. in inoculants made from presterilized peat. App. Environ. Microbiol. 1982, 44, 1246-1247. [CrossRef]

32. Nogales, J.; Domínguez-Ferreras, A.; Amaya-Gómez, C.V.; van Dillewijn, P.; Cuéllar, V.; Sanjuán, J.; Olivares, J.; Soto, M.J. Transcriptome profiling of a Sinorhizobium meliloti fadD mutant reveals the role of rhizobactin 1021 biosynthesis and regulation genes in the control of swarming. BMC Genomics 2010, 11, 157. [CrossRef]

33. Sahonero-Canavesi, D.X.; Sohlenkamp, C.; Sandoval-Calderón, M.; Lamsa, A.; Pogliano, K.; López-Lara, I.M.; Geiger, O. Fatty acid-releasing activities in Sinorhizobium meliloti include unusual diacylglycerol lipase. Environ. Microbiol. 2015, 17, 3391-3406. [CrossRef] [PubMed]

34. Fulda, M.; Heinz, E.; Wolter, F.P. The fadD gene of Escherichia coli K12 is located close to rnd at $39.6 \mathrm{~min}$ of the chromosomal-map and is a new member of the AMP-binding protein family. MGG 1994, 242, 241-249. [CrossRef] [PubMed]

35. Caviglia, J.M.; Li, L.O.; Wang, S.; DiRusso, C.C.; Coleman, R.A.; Lewin, T.M. Rat long chain acyl-CoA synthetase 5, but not 1, 2, 3, or 4, complements Escherichia coli fadD. J. Biol. Chem. 2004, 279, 11163-11169. [CrossRef] [PubMed]

36. Zhang, H.; Wang, P.; Qi, Q. Molecular effect of FadD on the regulation and metabolism of fatty acid in Escherichia coli. FEMS Microbiol. Lett. 2006, 259, 249-253. [CrossRef] [PubMed]

37. Nyström, T. Stationary-phase physiology. Annu. Rev. Microbiol. 2004, 58, 161-181. [CrossRef]

38. Farewell, A.; Diez, A.A.; DiRusso, C.C.; Nyström, T. Role of the Escherichia coli FadR regulator in stasis survival and growth phase-dependent expression of the uspA, fad, and fab genes. J. Bacteriol. 1996, 178, 6443-6450. [CrossRef]

39. Sauviac, L.; Philippe, H.; Phok, K.; Bruand, C. An extracytoplasmic function sigma factor acts as a general stress response regulator in Sinorhizobium meliloti. J. Bacteriol. 2007, 189, 4204-4216. [CrossRef]

40. Available online: http://iant.toulouse.inra.fr/bacteria/annotation/site/prj/rhime/doc/Bruand2007/ (accessed on 4 February 2020).

41. Campbell, J.W.; Morgan-Kiss, R.M.; E. Cronan, J., Jr. A new Escherichia coli metabolic competency: Growth on fatty acids by a novel anaerobic $\beta$-oxidation pathway. Mol. Microbiol. 2003, 47, 793-805. [CrossRef]

42. Lucas, R.L.; Lostroh, C.P.; DiRusso, C.C.; Spector, M.P.; Wanner, B.L.; Lee, C.A. Multiple factors independently regulate hilA and invasion gene expression in Salmonella enterica serovar Typhimurium. J. Bacteriol. 2000, 182, 1872-1882. [CrossRef]

43. Jamet, A.; Euphrasie, D.; Martin, P.; Nassif, X. Identification of genes involved in Neisseria meningitidis colonization. Infect. Immun. 2013, 81, 3375-3381. [CrossRef]

44. Banchio, C.; Gramajo, H.C. Medium- and long-chain fatty acid uptake and utilization by Streptomyces coelicolor A3(2): First characterization of a Gram-positive bacterial system. Microbiology 1997, 143, 2439-2447. [CrossRef] [PubMed]

45. Tejima, K.; Ishiai, M.; Murayama, S.O.; Iwatani, S.; Kajiwara, S. Candida albicans fatty acyl-CoA synthetase, CaFaa4p, is involved in the uptake of exogenous long-chain fatty acids and cell activity in the biofilm. Curr. Genet. 2018, 64, 429-441. [CrossRef] [PubMed]

46. Bullock, H.A.; Shen, H.; Boynton, T.O.; Shimkets, L.J. Fatty acid oxidation is required for Myxococcus xanthus development. J. Bacteriol. 2018, 200, e00572-17. [CrossRef] [PubMed]

47. Matsuoka, H.; Hirooka, K.; Fujita, Y. Organization and function of the YsiA regulon of Bacillus subtilis involved in fatty acid degradation. J. Biol. Chem. 2007, 282, 5180-5194. [CrossRef] [PubMed]

48. Black, P.N.; DiRusso, C.C. Yeast acyl-CoA synthetases at the crossroads of fatty acid metabolism and regulation. BBA-Mol. Cell Biol. Lipids 2007, 1771, 286-298. [CrossRef]

(C) 2020 by the authors. Licensee MDPI, Basel, Switzerland. This article is an open access article distributed under the terms and conditions of the Creative Commons Attribution (CC BY) license (http://creativecommons.org/licenses/by/4.0/). 